\title{
Rapid expansion of a saccular aneurysm on the left coronary sinus of Valsalva: a role for early surgical repair?
}

\author{
ROBERT T FAILLACE, PHILIP GREENLAND, NAVIN C NANDA* \\ From the Cardiology Unit, Department of Medicine, University of Rochester Medical Center, Rochester, New \\ York, USA
}

SUMMARY A thirty nine year old woman presented with ischaemic chest pain caused by a saccular aneurysm of the left coronary sinus of Valsalva. Over the next two weeks there was rapid dilatation of the aneurysm which led to left coronary artery compression and death from a massive myocardial infarction. It is suggested that early repair should be considered in patients with saccular aneurysm at this site, but only in the context of other management priorities.

We report a patient in whom expansion of a saccular aneurysm of the left coronary sinus of Valsalva over a few days caused direct compression of the left main coronary artery, myocardial infarction, and death due to cardiogenic shock. We wish to draw attention to the need for prompt diagnosis of this condition and to suggest that early surgical repair of sinus of Valsalva saccular aneurysm at this site should be considered since a delay of even a few days can prove fatal.

\section{Case report}

A 39 year old black woman was admitted to hospital for evaluation of symptoms of chest tightness. Weber-Christian disease had been diagnosed by skin biopsy 16 years previously. Nine months before admission she had been treated elsewhere for a breast abscess which yielded coagulase negative staphylococci when it was drained. Antibiotics had been given for 10 days. Chest $x$ ray examination at that time showed a left paraspinal mass at the T10 level which was shown by aortography to be a $2 \times 3$ centimetre saccular aneurysm of the posterolateral wall of the distal thoracic aorta. The aneurysm was resected and the aorta was repaired. Pathological examination showed atheromatous plaque and fibrin clot. Microbiological stains and blood cultures were not

Requests for reprints to Dr Philip Greenland, University of Rochester, Medical Center Box 679, 601 Elmwood Avenue, Rochester, New York 14642, USA.

*Present address: University of Alabama, Birmingham, Alabama, USA. requested. A Venereal Disease Research Laboratory test of her serum was negative.

When she was first admitted to our hospital cardiovascular examination showed blood pressure $110 / 70 \mathrm{~mm} \mathrm{Hg}$, pulse 80 beats per minute, with new II/VI ejection systolic and I/VI diastolic murmurs which were best heard along the left sternal border. There was mild normocytic, normochromic anaemia with an erythrocyte sedimentation rate of 150 Westergren units. Electrocardiography showed new first degree atrioventricular block. Real time cross sectional echocardiography demonstrated a large echo free space lateral to the aorta and a smaller space on the posterior aspect of the aorta which was consistent with aortic dissection or a sinus of Valsalva aneurysm, normal aortic valve leaflet, and normal left ventricular function (see Fig. 1). Pulsed Doppler examination showed active blood flow in the echo free space. Thoracic aortogram confirmed the presence of a saccular aneurysm $(2 \mathrm{~cm} \times 3 \mathrm{~cm})$ in the area of the posterior sinus of Valsalva with grade II aortic regurgitation. The coronary arteries were not displaced and were patent. We suspected infective endocarditis, but all six blood samples taken over a 48 hour period were culture negative. Elective operation was planned in two weeks time and the patient was discharged.

During the next week, she had several episodes of typical angina and was re-admitted. Electrocardiogram and chest $x$ ray examinations were unchanged but the diastolic murmur had become more intense (grade III/VI). The patient was treated with bed rest and anti-anginal drugs while awaiting 


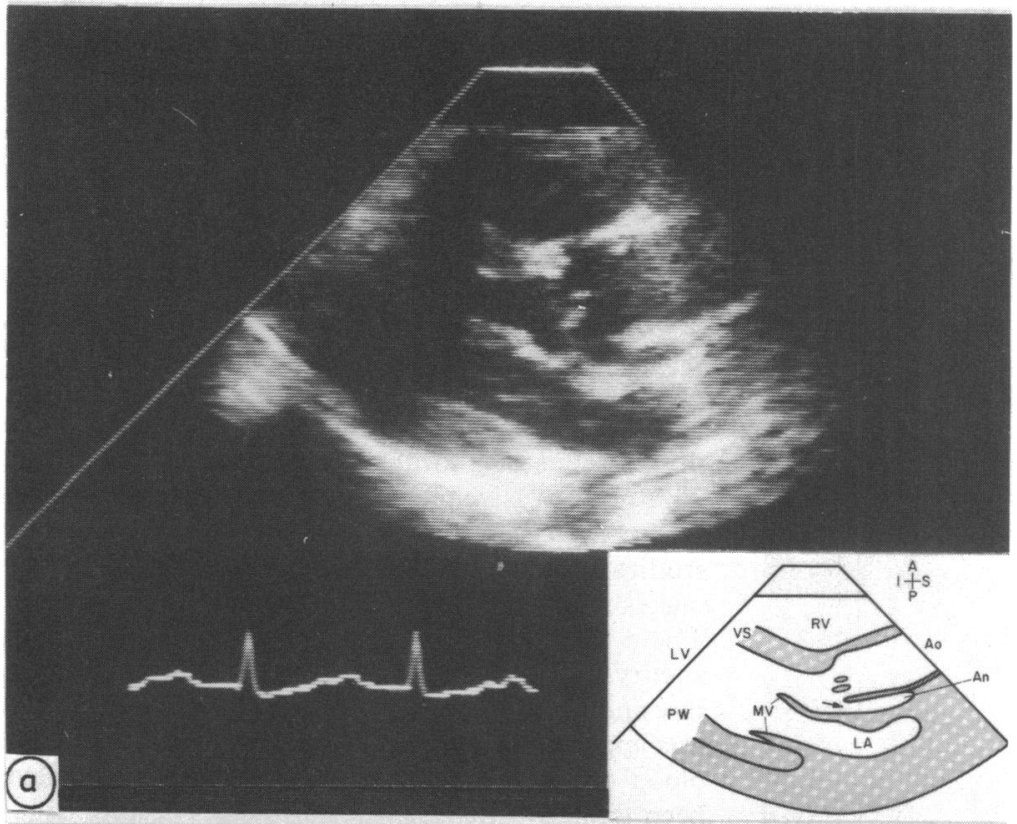

Fig. 1 Cross sectional echocardiograms in a patient with saccular aneurysm of the left coronary sinus of Valsalva. (a) The long axis view shows a linear echo free space (arrow) lying behind the posterior wall of the aorta ( $\mathrm{Ao}$ ) and communicating with its lumen. An, aneurysm; $L A$, left atrium; $L V$, left ventricle; $M V$, mitral valve; $P W$, left ventricular posterior wall; $R V$, right ventricle; $V S$, ventricular septum. (b) The short axis view at the level of the aortic valve ( $A V)$ demonstrates a large echo free space ( $A n$ ) consistent with aneurysm or dissection. Note the distortion of the aortic root produced by the aneurysm. $D A$, descending thoracic aorta; $L A$, left atrium; $R A$, right atrium; $T V$, tricuspid valve. Orientation symbols: $A$, anterior; $I$, inferior; $L$, left; $P$, posterior; $R$, right; $S$, superior.

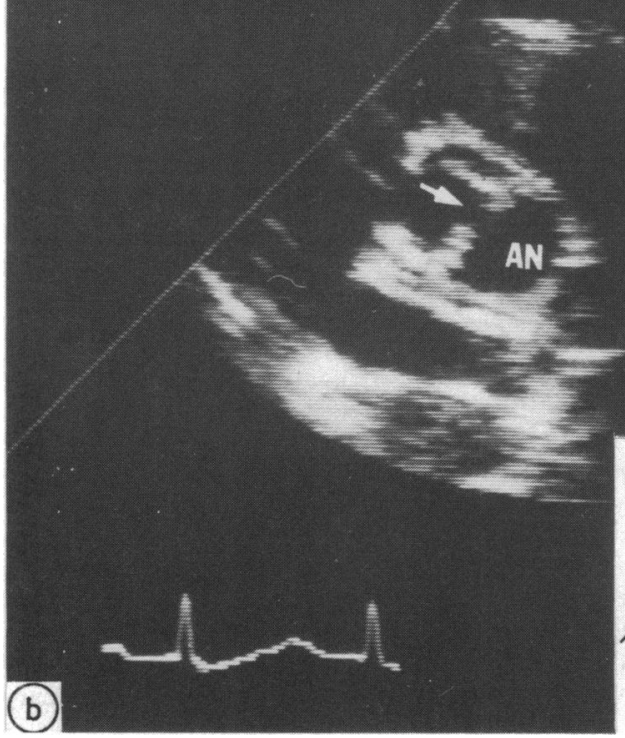

surgery. Four days later, she complained of severe substernal tightness lasting two hours; this was associated with a gallop rhythm, systemic arterial hypotension, and ST segment depression in leads I and V2-6. A repeat echocardiogram was not obtained but an emergency aortogram (Fig. 2) showed enlargement of the superior aspect of the sinus of Valsalva

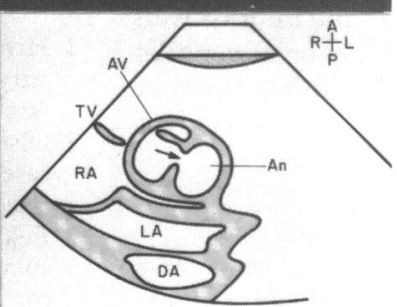

aneurysm, with displacement and compression of the left main coronary artery and grade III aortic regurgitation. At emergency operation the aneurysm was repaired, the disrupted aortic valve was excised and replaced, and a venous bypass graft was placed to the left anterior descending coronary artery. Shortly after operation she died from cardiogenic 


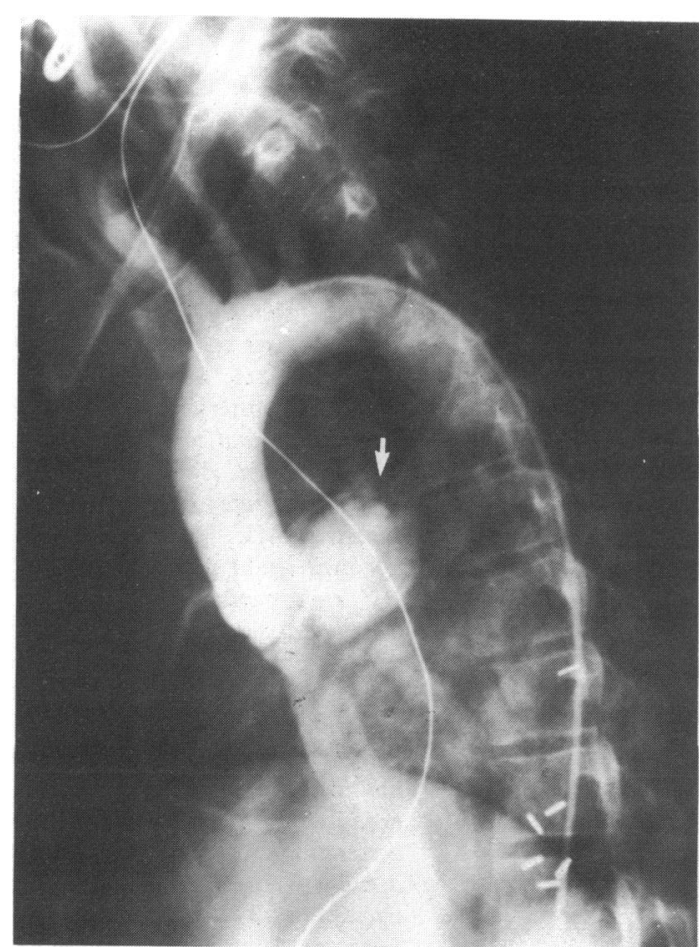

Fig. 2 Emergency aortogram showing enlarged sinus of Valsalva aneurysm superiorly displacing and occluding (arrow) the left main coronary artery. Aneurysm now measures $3 \times 3 \mathrm{~cm}$.

shock caused by an extensive myocardial infarction which had occurred before operation.

Warthin-Starry stained sections of the remnant of the sinus of Valsalva aneurysm, the aortic valve, and the previously resected distal thoracic aneurysm showed numerous rod shaped bacilli. Microscopical examination of the aortic valve and aneurysm showed an acute necrotising process with extensive tissue destruction and an abscess in the inferior wall of the aneurysm. There was no evidence of syphilis or cystic medial necrosis. Blood and tissue taken at necropsy were culture negative.

\section{Discussion ·}

We believe that this case demonstrates that early repair should be considered in patients with symptoms of myocardial ischaemia due to saccular aneurysm of a coronary sinus of Valsalva. Although there have been other reports of myocardial infarction caused by coronary artery compression from such aneurysms, $!^{-3}$ our case illustrates that aneurysmal enlargement can occur within weeks of initial presentation and can be rapidly fatal. Because it was our impression that the patient's condition was fairly stable, we and our surgical colleagues did not initially regard operation as urgent. This report is the first to describe the rapidity with which fatal complications can occur in a patient with saccular aneurysm of the left coronary sinus of Valsalva, and we believe that, depending on the clinical context, early surgical repair should be considered in similar cases. Patients with infective endocarditis may present with various difficult management problems, as well as saccular aneurysm of a coronary sinus, and priorities must be established for each case.

In our patient echocardiography first alerted us to the presence of a localised aneurysm or dissection. This non-invasive technique as well as being of diagnostic value can also be used for serial follow up studies without any risk to the patient. Its portability makes it an ideal bedside tool in acutely ill patients. There are two other noteworthy points, first, aneurysmal dilatation of a single coronary sinus is rare and usually caused by infective endocarditis, ${ }^{4}$ as was apparent in our patient despite several negative blood cultures. Our patient should probably have been treated-with systemic antibiotics despite negative cultures. Secondly, although our patient had Weber-Christian disease, we know of no reported association of this' syndrome with sinus of Valsalva aneurysm and we do not believe there was a causal relation in our patient. ${ }^{5}$

Dr Faillace's work was supported by a grant from the National Heart, Lung, and Blood Institute for research training in cardiology. Dr Greenland is a teaching and research scholar of the American College of Physicians and a preventive cardiology academic awardee of the National Heart, Lung, and Blood Institute.

\section{References}

1 Hiyamuta K, Ohtsuki T, Shimamatsu M, et al. Aneurysm of the left aortic sinus causing myocardial infarction. Circulation 1983; 67: 1151-4.

2 Olsen J. Aneurysm of the aortic sinus of Valsalva. A case of rupture and myocardial infarction. Acta Pathologica et Microbiologica Scandinavica 1969; 76: 12-8.

3 Feldman R, Buchoff H, Pepine CJ, Conti CR. Aneurysm of sinus of Valsalva. Cause of dynamic coronary constriction after aortic valvular replacement and bacterial endocarditis. Chest 1978; 74: 312-4.

4 Buchbinder NA, Roberts WC. Left sided valvular active infective endocarditis. A study of forty-five necropsy patients. $A m$ f Med 1972; 53: 20-35.

5 Lazarus G. Panniculitis and disorders of the subcutaneous fat. In: Wyngaarden JB, Smith LH, eds. Cecil textbook of medicine: Philadelphia: WB Saunders, 1982: 1898-9. 